\title{
The presence of high level soluble herpes virus entry mediator in sera of gastric cancer patients
}

\author{
Sook-Kyoung Heo', Seong-A Ju${ }^{3}$, Gyu Yeol Kim², \\ Sang-Min Park', Sung Hun Back', \\ Neung-Hwa Park', Young Joo Min ${ }^{3}$, Won G. $\mathrm{An}^{4}$, \\ Thu-Ha Thi Nguyen ${ }^{1}$, Sun-Min Kim ${ }^{1}$ \\ and Byung-Sam Kim ${ }^{1,5}$ \\ ${ }^{1}$ Department of Biological Sciences \\ University of Ulsan \\ Ulsan 680-749, Korea \\ ${ }^{2}$ Department of Surgery \\ Ulsan University Hospital \\ University of Ulsan College of Medicine \\ ${ }^{3}$ Biomedical Research Center \\ Ulsan University Hospital \\ University of Ulsan \\ Ulsan 682-714, Korea \\ ${ }^{4}$ School of Korean Medicine \\ Pusan National University \\ Pusan 626-870, Korea \\ ${ }^{5}$ Corresponding author: Tel, 82-52-259-2350; \\ Fax, 82-52-259-2740; E-mail, bskim @mail.ulsan.ac.kr \\ http://dx.doi.org/10.3858/emm.2012.44.2.010
}

Accepted 23 November 2011

Available Online 24 November 2011

Abbreviations: DCF-DA, 2',7'-dichlorofluorescin diacetate; HVEM, herpes virus entry mediator; LIGHT, homologous to lymphotoxins, shows inducible expression and competes with herpes simplex virus glycoprotein $D$ for herpes virus entry mediator (TNF Ligand Superfamily, Member 14; TNFSF14); rhLIGHT, recombinant human LIGHT

\begin{abstract}
The development of gastric cancer (GC) is closely related to chronic inflammation caused by Helicobacter pylori infection, and herpes virus entry mediator (HVEM) is a receptor expressed on the surface of leukocytes that mediates potent inflammatory responses in animal models. However, the role of HVEM in human GC has not been studied. Previously, we showed that the interaction of HVEM on human leukocytes with its ligand LIGHT induces intracellular calcium mobi-
\end{abstract}

lization, which results in inflammatory responses including induction of proinflammatory cytokine production and anti-bacterial activities. In this study, we report that leukocytes from GC patients express lower levels of membrane HVEM (mHVEM) and have lower LIGHT-induced bactericidal activities than those from healthy controls (HC). In contrast, levels of soluble HVEM (sHVEM) in the sera of GC patients were significantly higher than in those of HC. We found that monocyte membrane-bound HVEM is released into the medium when cells are activated by proinflammatory cytokines such as TNF- $\alpha$ and IL-8, which are elevated in the sera of GC patients. mHVEM level dropped in parallel with the release of SHVEM, and release was completely blocked by the metalloprotease inhibitor, GM6001. We also found that the low level of mHVEM on GC patient leukocytes was correlated with low LIGHT-induced bactericidal activities against $H$. pylori and $S$. aureus and production of reactive oxygen species. Our results indicate that mHVEM on leukocytes and SHVEM in sera may contribute to the development and/or progression of GC.

Keywords: cytokines; inflammation; monocytes; receptors, tumor necrosis factor, member 14; stomach neoplasms; TNFSF14 protein, human

\section{Introduction}

It is now well established that inflammation plays critical roles in the development of cancer and metastases. Inflammation is caused by many agents including microbial infections, autoimmune diseases and obesity, and involves complex interactions between proinflammatory cytokines and cells (Mantovani et al., 2008). Tumor necrosis factor $\alpha(T N F-\alpha)$ is one of the most potent proinflammatory cytokines and is involved in the pathogenesis of many inflammatory diseases and tumors (Balkwill, 2009). Therefore, TNF- $\alpha$ and its receptors have long been targets in the treatment of a variety of human diseases. For example, some patients with inflammatory diseases are helped by preventing TNF- $\alpha$ from binding to TNF receptors 
(TNFR). However, some patients are resistant to treatment by TNF- $\alpha$ blockade, implying that the role of TNF- $\alpha$ is not universal in human diseases (Zhu et al., 2010; Benucci et al., 2011). The TNF superfamily (TNFSF) and the TNFR superfamily (TNFRSF) each contain several members, and TNFSF-TNFRSF interactions regulate a number of normal and pathological processes, including immune responses, hematopoiesis, morphogenesis, tumorigenesis, septic shock, and inflammatory disease (Aggarwal, 2003). It is likely that additional TNFSF-TNFRSF interactions are involved in inflammation-induced human diseases including those resistant to TNF- $\alpha$ blockade.

Herpes virus entry mediator (HVEM), a TNFRSF protein, is widely expressed on the plasma membranes of hematopoietic lineage cells, such as $T$ cells, B cells, natural killer cells, monocytes, neutrophils and dendritic cells (Kwon et al., 1997; Heo et al., 2006). HVEM has multiple ligands and is unique among TNFRSF proteins in binding to both TNFSF and immunoglobulin superfamily (IGSF) proteins. In addition to the TNFSF proteins LIGHT (homologous to lymphotoxins, shows inducible expression and competes with herpes simplex virus glycoprotein D for HVEM, a receptor expressed by $T$ lymphocytes) and lymphotoxin $\alpha$, the IGSF proteins $B$ and T lymphocyte attenuator (BTLA) and CD160 bind to HVEM (Shui et al., 2011), as does herpes simplex virus glycoprotein D. When LIGHT binds to HVEM, positive co-stimulatory signals are delivered through both LIGHT and HVEM that result in potent leukocyte inflammatory responses, such as the production of inflammatory cytokines and ROS. On the other hand, binding of HVEM to BTLA delivers inhibitory signals by recruiting tyrosine phosphatase, and these signals lead to suppression of immune responses. Therefore, HVEM can act as a molecular switch, either activating or inhibiting immune responses. In this regard, many studies have focused on the HVEM/LIGHT/BTLA pathway in animal models of tumors, autoimmune disease, infection and transplantation (del Rio et al., 2010). However, few studies have investigated the roles of HVEM and/or LIGHT in human diseases including cancer.

Gastric cancer (GC) is the second leading cause of cancer-related death worldwide, and approximately 700,000 people succumb to it each year. It has been shown that Helicobacter pylori, by which half the world's population is infected, is closely associated with the development of GC, suggesting that inflammation is involved in that process (Wroblewski et al., 2010). In a previous study, we showed that HVEM induces potent inflammatory responses by neutrophils and monocytes (Heo et
Table 1. Gastric Cancer Patient Characteristics

\begin{tabular}{cc}
\hline Clinopathological Characteristics & No \\
\hline Age & 5 \\
$<60$ & 10 \\
$>60$ & \\
Sex & 6 \\
Male & 9 \\
Female & \\
Cancer stage ${ }^{\mathrm{a}}$ & 5 \\
IA & 3 \\
IB & 1 \\
IIA & 3 \\
IIB & 1 \\
IIIA & 2 \\
IIIC & \\
Gastritis & \\
Positive & 6 \\
Negative & 9 \\
H. pyroli infection & \\
Positive & 11 \\
Negative & 3 \\
ND &
\end{tabular}

${ }^{a}$ TNM stage; ${ }^{b}$ determined by endoscopy; ${ }^{c}$ determined by CLO test; ${ }^{d}$ not determined

al., 2006). Therefore, we hypothesized that leukocytes from GC patients might have altered levels of HVEM and HVEM-mediated immune responses. Here we report that the leukocytes of GC patients contain significantly lower levels of HVEM than those of healthy controls, and GC patients have higher soluble HVEM concentrations in their sera than do healthy controls. Surprisingly, we also found that membrane-bound HVEM is shed when cells are activated by a variety of stimuli including the proinflammatory cytokines IL- 8 and TNF- $\alpha$, whose concentrations are highly elevated in the sera of GC patients.

\section{Results}

\section{Patient characteristics}

A summary of the enrolled patients is presented in Table 1. Blood samples from 15 patients with GC and 22 healthy controls $(\mathrm{HC})$ were investigated. Median age of GC and $\mathrm{HC}$ was $59.5 \pm 11.4$ and $53.9 \pm 2.7$ years, respectively. 


\section{GC patient leukocytes have reduced levels of membrane HVEM (mHVEM)}

It has previously been demonstrated by this research group that HVEM was expressed on the membranes of T-cells, B-cells, monocytes and neutrophils isolated from human peripheral blood (Heo et al., 2006). Of those, monocytes and neutrophils were the most highly HVEM-expressing cells. HVEM expression on the membranes of monocytes and neutrophils in GC patients and healthy controls $(\mathrm{HC})$ were compared by staining cells with fluorescence-labeled anti-HVEM antibody and examining them by FACS. As shown in Figure 1A, GC patient monocytes had significantly lower levels of $\mathrm{mHVEM}$ than $\mathrm{HC}$ monocytes. When analyzed as the percentage of HVEM-expressing cells, $94.6 \pm 3.5 \%$ of $\mathrm{HC}$ monocytes expressed HVEM, compared with only $49.1 \pm 12.8 \%$ of GC patient monocytes (Figure 1B). The percentage of HVEM-expressing neutrophils was also substantially lower in GC patients than in $\mathrm{HC}(37.0 \pm$ $9.5 \%$ vs $67.7 \pm 10.7 \%)$.

It has been shown that soluble HVEM (sHVEM) is elevated in patients with allergic and autoimmune diseases such as allergic asthma, atopic dermatitis and rheumatoid arthritis (Jung et al., 2003). However, sHVEM levels in cancer patients has not been reported. Blood levels of SHVEM in $\mathrm{HC}$ and GC patients were determined by ELISA as described in Methods. In sharp contrast with the results for membrane-bound HVEM, sHVEM levels in GC patient sera were more than 3-fold higher than in $\mathrm{HC}$ (mean $\pm \mathrm{SD} ; 748 \pm 461$ and 2,619 \pm $1,195 \mathrm{pg} / \mathrm{ml}$ for $\mathrm{HC}$ and $\mathrm{GC}$ patients, respectively $(P<0.01)$ (Figure 1C).

\section{High levels of proinflammatory cytokines in GC patient sera}

Cytokines are major regulators of immune cells affecting receptor expression and cell activation. It has been reported that sera of advanced stage GC patients contain elevated levels of inflammatory cytokines (Tsujimoto et al., 2010). Serum cytokine levels were measured to investigate mechanisms underlying altered expression of leukocyte mHVEM and sHVEM in GC patients. As shown in Figure 2, TNF- $\alpha$, IL-1 $\beta$, IL-6, and IL-8 levels were significantly higher in the sera of GC patients than in those of HC. In contrast, IL-2, IL-4, IL-10 and IFN- $\gamma$ levels were significantly lower, while IL-12 levels did not differ significantly in GC patients and $\mathrm{HC}$.
A
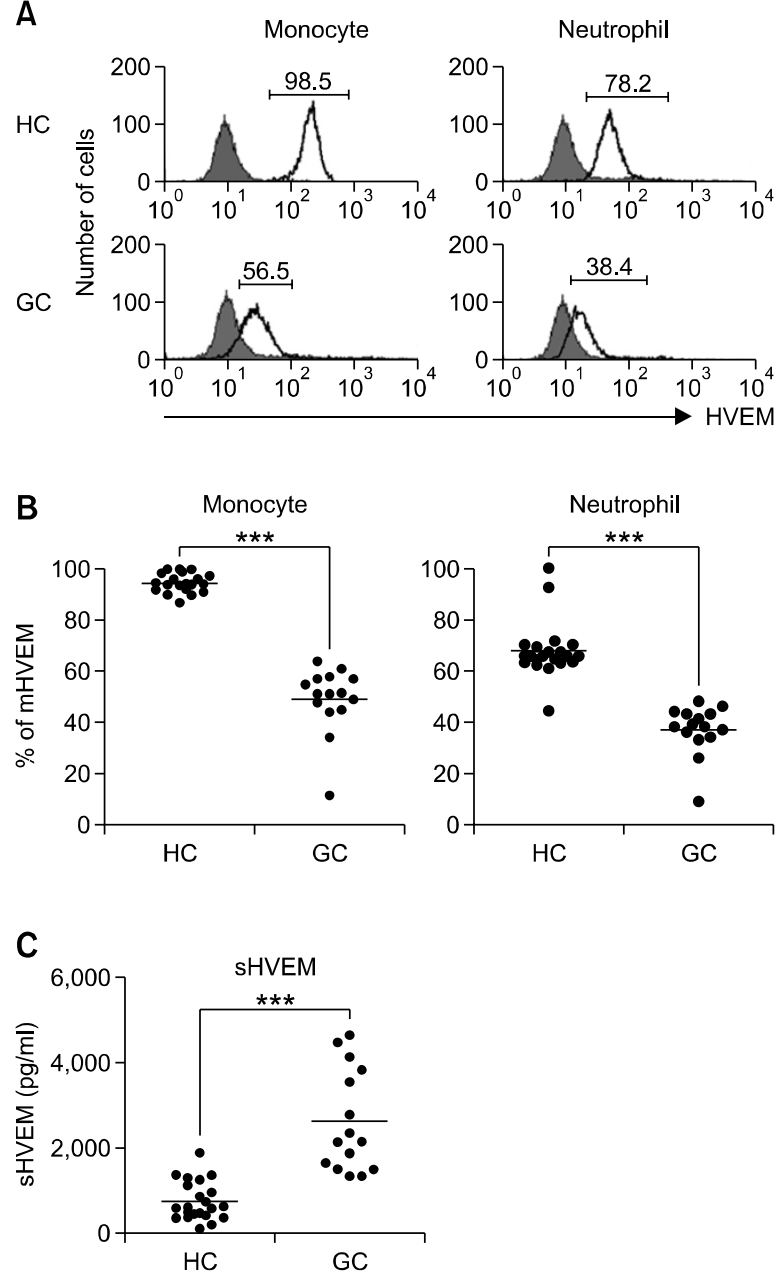

Figure 1. GC patients have lower mHVEM levels in their leukocytes and higher sHVEM levels in their sera than healthy controls. (A) Histogram of mHVEM expression in monocytes and neutrophils. Monocytes and neutrophils of GC patients and healthy controls were purified as described in Methods. Cells were stained with FITC-conjugated anti-HVEM antibody (open histogram) or isotype control (filled histogram) and analyzed by FACS. Results shown are representative of three experiments. (B) Percentages of HVEM-expressing monocytes (left panel) and neutrophils (right panel) in $\mathrm{GC}$ patients and $\mathrm{HC}$ were determined as in A. Each dot represents the \% of HVEM expressing cells in a sample. Horizontal bars depict means. (C) sHVEM levels in sera of GC patients and HC. Concentrations of SHVEM in sera were determined by ELISA as described in Methods. Horizontal bars depict means. Each datum shown is the mean of triplicate measurements. ${ }^{* * *} P<0.001$ compared with control groups.

\section{Stimulation of monocytes induces release of SHVEM with a concurrent decrease of MHVEM}

It has been shown that membrane-associated HVEM on T lymphocytes is downregulated when the cells are activated (Morel et al., 2000). However, the regulation of HVEM expression on leukocytes has not been reported. It was hypothesized that activation of leukocytes by inflammatory stimulants might induce mHVEM release into the extracellular 

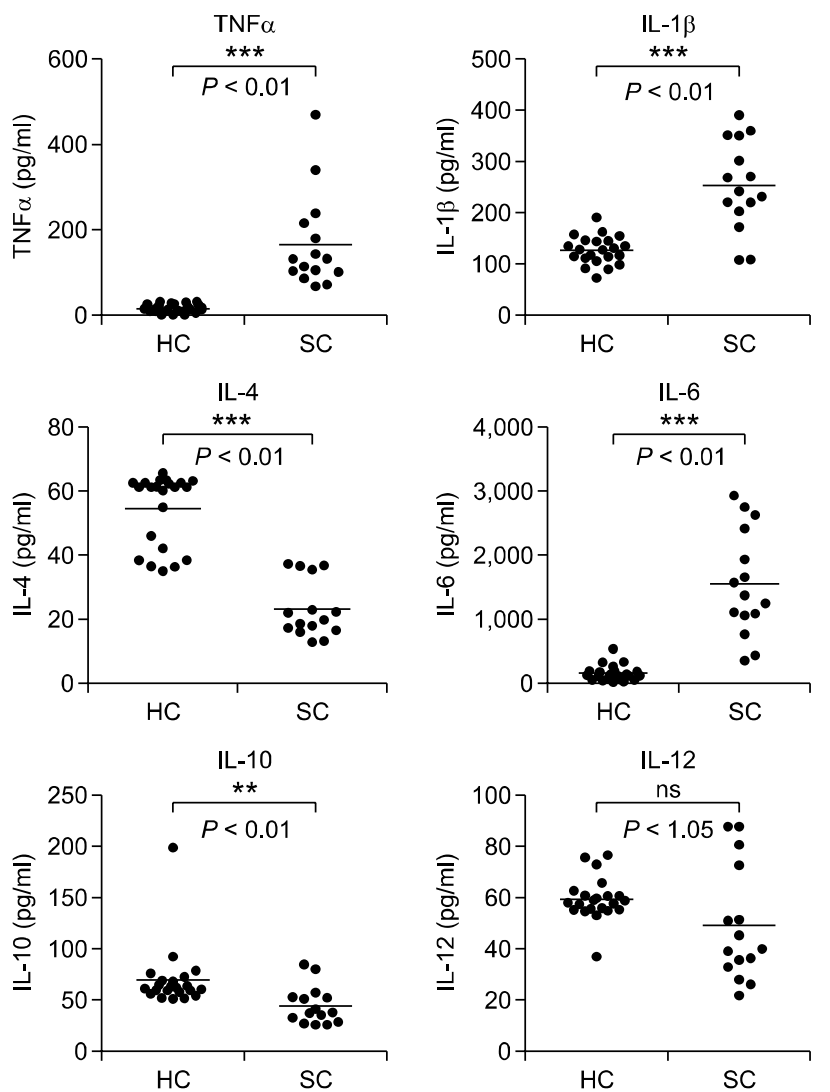

space and lead to the high levels of sHVEM observed in GC patient sera. To test this idea, monocytes were cultured in the presence of a variety of cell activating agents, such as LPS, PHA, calcium ionophore A23187 and PMA for $24 \mathrm{hr}$ and measured levels of HVEM on cell membranes (mHVEM), and of sHVEM in culture supernatants. As shown in Figure $3 \mathrm{~A}$, all of those stimulants decreased $\mathrm{mHVEM}$ and increased sHVEM, indicating that non-specific activation of monocytes induces release of mHVEM into the culture medium. The specific HVEM ligand, rhLIGHT, also dose-dependently decreased mHVEM and increased sHVEM (Figure 3B). Since GC patients have high levels of systemic inflammatory cytokines, such as TNF- $\alpha$ and IL-8 (Figure 2), it was investigated whether cytokines also affected mHVEM expression on monocytes and sHVEM levels. As expected, both cytokines decreased $\mathrm{mHVEM}$ and increased SHVEM in the culture medium (Figure 3C).

The release of extracellular protein domains, called ectodomain shedding, is now recognized as a general mechanism for regulating the function of transmembrane proteins. Zinc-based metalloproteases, such as metalloprotease disintegrins and matrix metalloproteases, mediate the shedding of a variety of membrane receptors and cytokines
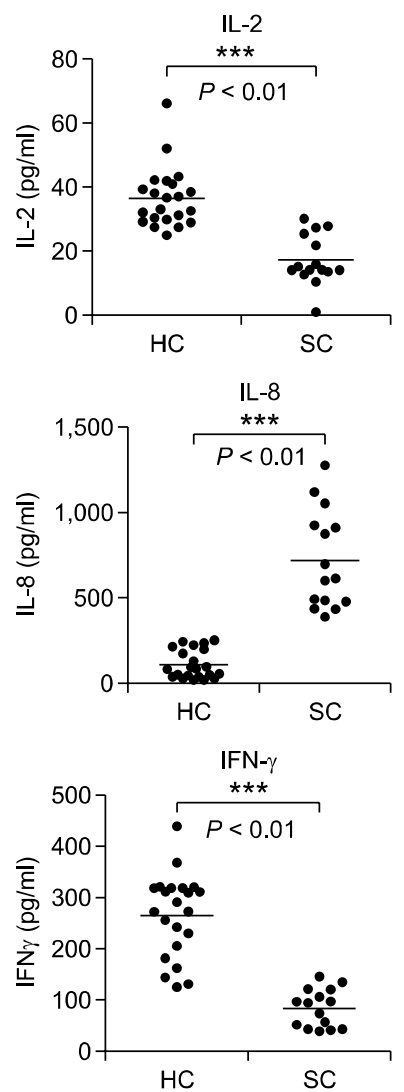

Figure 2. Cytokine levels in sera of GC patients and HC. Peripheral blood from GC patients and $\mathrm{HC}$ was coagulated to obtain sera. Cytokine concentrations in sera were determined with ELISA kits (Endogen, $M A)$. Each datum is the mean of triplicate measurements. Horizontal bars depict means. ${ }^{* *} P<0.01$ and ${ }^{* * *} P<0.001$ compared with $\mathrm{HC}$ ns, not significant.
(Arribas and Borroto, 2002). To see whether metalloproteases are involved in the activation-induced release of sHVEM in leukocytes, the effect of GM6001, which inhibits a wide spectrum of metalloproteases, was studied. Freshly isolated human monocytes from $\mathrm{HC}$ were stimulated with rhLIGHT, rhIL-8 or rhTNF- $\alpha$ in the presence of GM6001 for $24 \mathrm{hr}$ and mHVEM and sHVEM levels in the culture medium were determined. It was found that GM6001 progressively inhibited the rhLIGHT-induced decrease of MHVEM and rhLIGHT-mediated increase of sHVEM (Figure 4A). Release of monocyte mHVEM by rhIL-8 and rhTNF- $\alpha$ was also inhibited by GM6001 (Figure 4B). These results indicate that when monocytes are activated, mHVEM is cleaved by a metalloprotease and released into the culture medium.

\section{GC patient leukocytes have lower HVEM-mediated bactericidal activities and produce less ROS than leukocytes of healthy controls}

Our finding that mHVEM is low on GC leukocytes prompted a test of whether altered mHVEM level affected HVEM-mediated leukocyte function. It was previously demonstrated by this research group that binding of recombinant human LIGHT (rhLIGHT) 
A
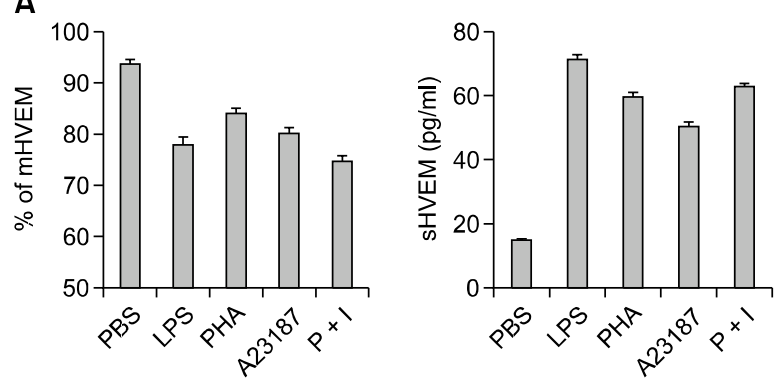

B

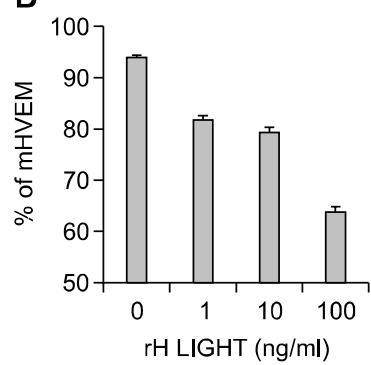

C
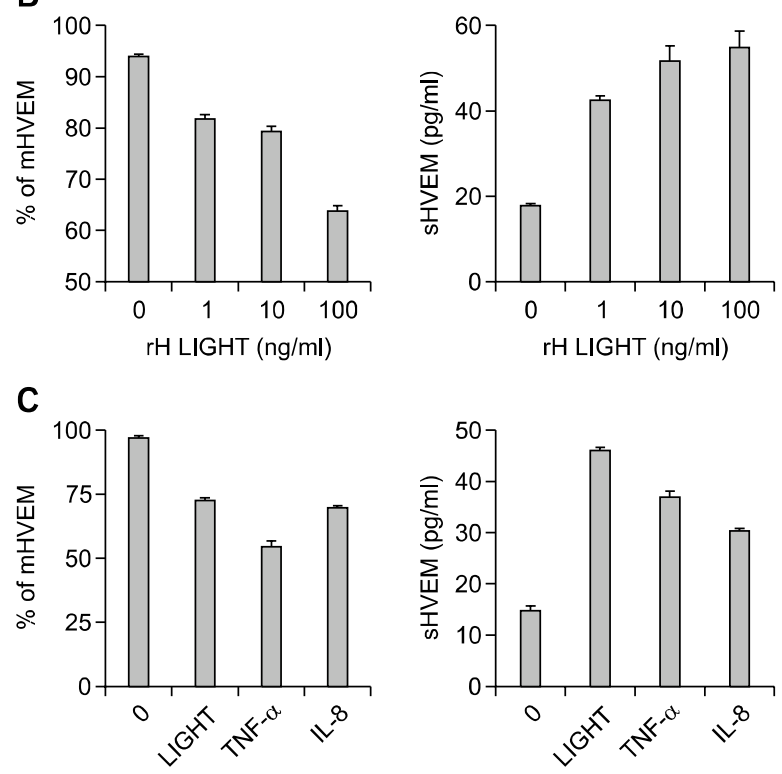

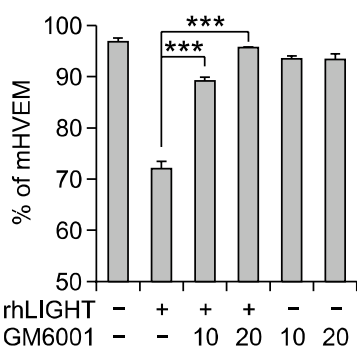

Figure 3. Stimulation of monocytes decreases $\mathrm{mHVEM}$ and increases sHVEM. (A) Monocytes from HC were cultured in RPMI1640 + 10\% FBS

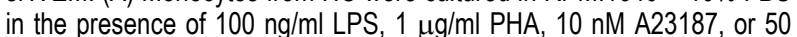
$\mathrm{ng} / \mathrm{ml}$ PMA and $200 \mathrm{ng} / \mathrm{ml}$ ionomycin. After $24 \mathrm{hr}$, cells and culture supernatants were harvested. Cells were stained with anti-HVEM-FITC mAbs or isotype control mAbs (Mouse lgG1) and analyzed by flow cytometry, and percentages of mHVEM-expressing cells are shown (left panel). Concentrations of sHVEM in culture supernatants were analyzed by ELISA (right panel). (B) Monocytes were cultured in the presence of 0,1 , 10 or $100 \mathrm{ng} / \mathrm{ml} \mathrm{rhLIGHT}$ for $24 \mathrm{hr}$, and the $\%$ of mHVEM-expressing cells and sHVEM concentrations in culture supernatants were determined as in (A). (C) Monocytes were cultured with $100 \mathrm{ng} / \mathrm{ml}$ of rhLIGHT, rhTNF- $\alpha$ or rhlL- 8 for $24 \mathrm{hr}$, and the $\%$ of mHVEM expressing cells and SHVEM concentrations in culture supernatants were determined as in (A). Results shown are representative of at least three independent experiments and are means \pm SEM of triplicates. ${ }^{* * *} P<$ 0.001 compared with rhLIGHT- alone group. mHVEM, membrane bound HVEM; sHVEM, soluble HVEM.

protein to HVEM in leukocytes activates cytoplasmic phospholipase $\gamma 1$, which results in ROS generation and enhancement of bactericidal activities (Heo et al., 2006, 2007). Neutrophils and monocytes from $\mathrm{HC}$ and $\mathrm{GC}$ patients were isolated, allowed to ingest $S$. aureus and incubated with 1, 10, and $100 \mathrm{ng} / \mathrm{ml}$ of $\mathrm{rhLIGHT}$ for $10 \mathrm{~min}$; then the number of viable bacteria remaining in the cells

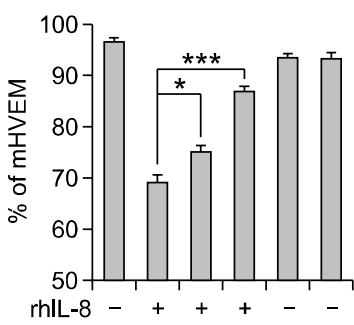

GM6001 - -10201020
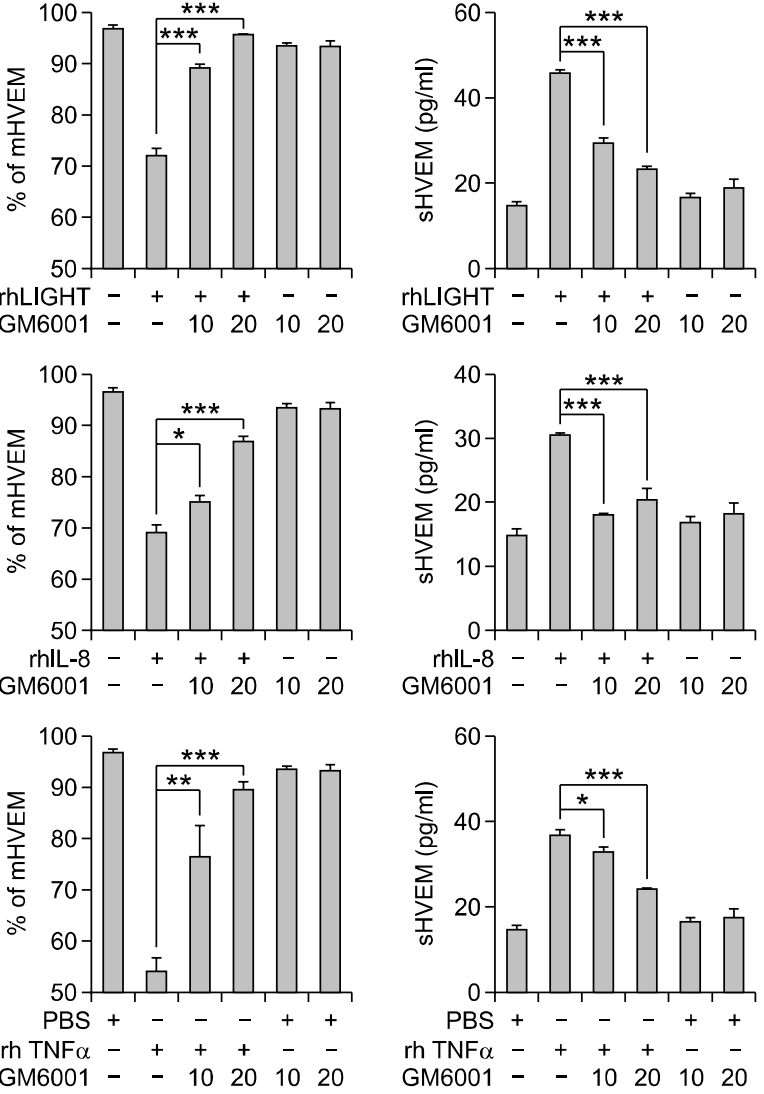

Figure 4. Metalloprotease inhibitor GM6001 blocks the activation-induced decrease of mHVEM and increase of sHVEM in cultured monocytes. Cells purified from $\mathrm{HC}$ were cultured in the presence of the indicated agonists (10 ng/ml) and/or GM6001 (10 or $20 \mu \mathrm{M})$, and the \% of $\mathrm{mHVEM}$ expressing cells and SHVEM concentrations in culture supernatants were determined as in Figure 3. Results shown are representative of at least three independent experiments and are means \pm SEM of triplicates. ${ }^{* * *} P<0.001,{ }^{* *} P<0.01$ and, ${ }^{\star} P<0.05$ compared with rhLIGHT alone group. mHVEM, membrane bound HVEM; sHVEM, soluble HVEM.

was determined by plating serial tenfold dilutions of cell lysates on agar plates. Figure $5 \mathrm{~A}$ shows that the bactericidal activity of $\mathrm{HC}$ monocytes and neutrophils was dose-dependently increased by rhLIGHT. The bactericidal activities of GC patient monocytes and neutrophils were also increased by rhLIGHT but to a lesser extent. Heat-inactivated rhLIGHT did not enhance the bacterial killing activity of monocytes and neutrophils, indicating that there was no contamination with endotoxins (Figure $5 A)$.

Next, rhLIGHT-induced bactericidal activities of GC patient monocytes and neutrophils against $H$. pylori, which is considered as a major agent developing gastric cancer, was determined. As shown in Figure 5B, $H$. pylori killing activities of GC patient monocytes and neutrophils were significantly lower 
A
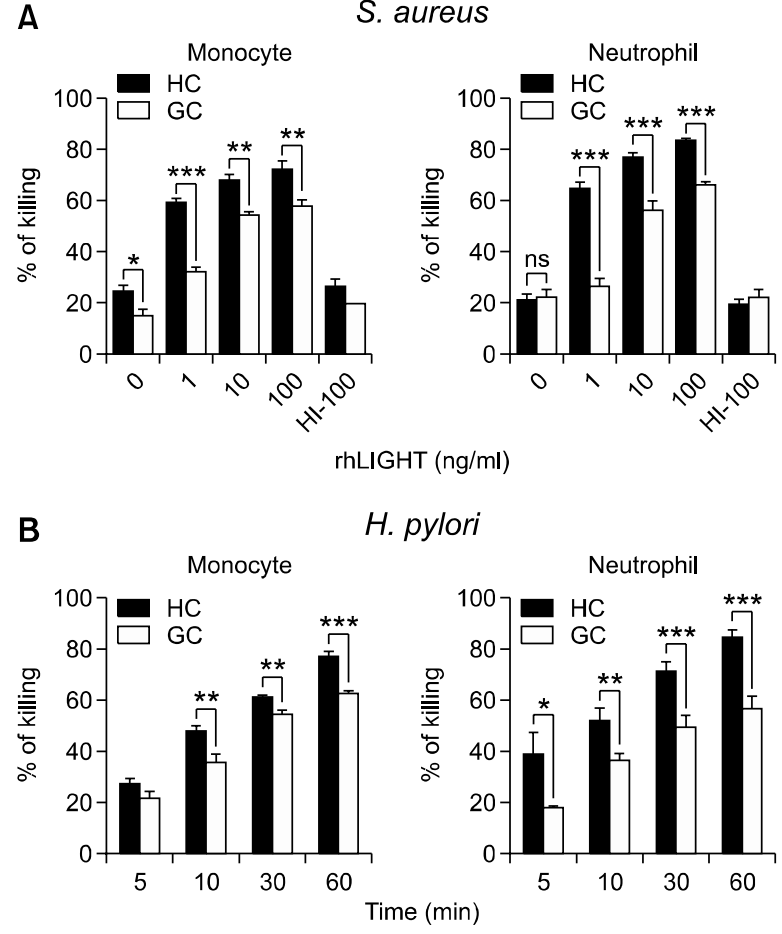

C

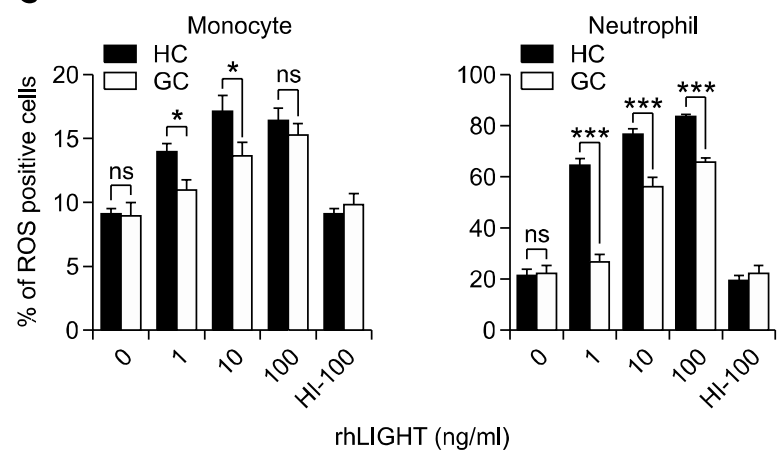

Figure 5. HVEM-mediated bacterial killing and ROS production by GC patient leukocytes are lower than by $\mathrm{HC}$ leukocytes. Monocytes and neutrophils of GC patients and healthy controls were purified and incubated with opsonized S. aureus or $\mathrm{H}$. pylori for $3 \mathrm{~min}$. After washing to remove non-ingested bacteria, $(A)$ cells were incubated for 10 min with indicated concentrations of rhLIGHT or (B) cells were treated with $10 \mathrm{ng} / \mathrm{ml}$ of rhLIGHT for indicated times. After incubation, cells were lysed in distilled water, and numbers of viable bacteria per million cells were determined by plating on agar. (C) HVEM-mediated ROS production. Cells were stained with the ROS-sensitive fluorescent dye, DCF-DA, and cultured with $0,1,10$ or $100 \mathrm{ng} / \mathrm{ml}$ of rhLIGHT. After $45 \mathrm{~min}$, they were harvested and percentages of ROS-positive cells were assessed by flow cytometry. Filled bar, healthy controls; open bar, GC patients. HI-100, heat-inactivated rhLIGHT $100 \mathrm{ng} / \mathrm{ml}$. Results shown are representative of at least three independent experiments from different blood donors and are means \pm SEM. ${ }^{*} P<0.05 ;{ }^{* *} P<0.01$ and ${ }^{* * *} P<0.001$ compared with control group.

than those of $\mathrm{HC}$ cells. These results indicate that GC patient leukocytes have reduced activity of HVEM-mediated clearance of $H$. pylori infection in
GC patients, which might aggravate inflammation associated with $\mathrm{GC}$ development.

ROS, which are produced by activated, phagosome-bound NADPH oxidases, are major component of the bactericidal activity of monocytes and neutrophils and the rhLIGHT-HVEM interaction stimulates ROS production in human monocytes and neutrophils (Segal, 2005). rhLIGHT-induced ROS generation was compared in the leukocytes of $\mathrm{GC}$ patients and $\mathrm{HC}$. Cells were stained with the ROS-sensitive fluorescent dye DCF-DA and incubated with rhLIGHT. They were then harvested, and fluorescent cells were detected by flow cytometry. As shown in Figure 5B, exposure of $\mathrm{HC}$ monocytes and neutrophils to rhLIGHT ( 1 and 10 $\mathrm{ng} / \mathrm{ml}$ ) stimulated ROS production, but $100 \mathrm{ng} / \mathrm{ml}$ rhLIGHT had less effect. rhLIGHT treatment of GC patient monocytes and neutrophils also increased ROS production. However, the \% of ROS positive GC patient leukocytes was significantly lower than in $\mathrm{HC}$ leukocytes when compared at the same rhLIGHT concentrations, except for neutrophils treated with $100 \mathrm{ng} / \mathrm{ml}$ of rhLIGHT, which was maximally effective in stimulating ROS production. These results together with our previous finding that rhLIGHT-mediated bactericidal activity in leukocytes is dependent on ROS generation (Heo et al., 2006), suggest that mHVEM levels on leukocytes influence HVEM-mediated leukocyte functions.

\section{Discussion}

Ectodomain shedding regulates the activity of a number of transmembrane proteins. Several physiological agents, such as chemokines, cytokines, growth factors and non-specific agonists such as PMA, have been shown to induce ectodomain shedding (Arribas and Borroto, 2002). TNFSF and TNFRSF proteins have also been shown to be regulated by ectodomain shedding. For example, TNF- $\alpha$ is synthesized in a transmembrane form, proTNF- $\alpha$, and cleavage by TNF- $\alpha$ converting enzyme (TACE; also known as ADAM17), ADAM10 or MMP-7, releases the receptor-binding domain into the extracellular space (Wong et al., 1989; Hikita et al., 2009). Ectodomain shedding of HVEM has not been previously reported. Our earlier finding that patients with autoimmune disease have high levels of SHVEM in their sera is the first report of the existence of sHVEM (Jung et al., 2003). We observed that levels of SHVEM in sera of patients with inflammatory diseases, such as rheumatoid arthritis, atopic dermatitis, psoriasis, systemic lupus erythematosus, Bechet's disease, allergic asthma or pneumonia, were 2- to 5-fold higher 
than in sera of healthy controls. In the present study, we found that GC patients have roughly 3-fold higher levels of sHVEM levels in their sera than in age-matched healthy controls. Although we did not demonstrate cleavage of HVEM, several observations suggest that the SHVEM is produced by ectodomain shedding rather than by simple secretion. The primary feature of ectodomain shedding is that it is induced by non-specific agents such as PMA and many agonists (Arribas and Borroto, 2002), and we showed that the same was true for the increase of SHVEM and decrease of mHVEM in cultured monocytes (Figure 3A). Second, GC patients have high levels of inflammatory cytokines (Figure 2) and these were shown to induce the increase of sHVEM and decrease of mHVEM in cultured monocytes (Figure 3C). Lastly, inhibition of a metalloprotease known to cause ectodomain shedding also inhibited the release of sHVEM by cultured monocytes (Figure 4).

The soluble domains of receptors generated by shedding can regulate ligand functions by inhibiting or promoting the formation of active signaling complexes (Rose-John and Heinrich, 1994). Furthermore, transmembrane and cytoplasmic domains that remain bound to the cell following receptor ectodomain shedding have been shown in some cases to have autonomous signaling abilities (Fortini, 2001). At present, the roles of SHVEM and the transmembrane/cytoplamic domain of HVEM are completely unknown, although the signaling pathway of mHVEM is well defined: when LIGHT binds to mHVEM, there is intracellular mobilization of calcium ion, and binding of adaptor proteins, referred to as TNFR-associated factors, to the cytoplasmic domain of HVEM leads to NF $\kappa B$ activation (Wallach et al., 1999; Heo et al., 2007). This process can result in costimulation of $T$ cells (Tamada et al., 2000), induction of dendritic cell maturation (Zou et al., 2004), stimulation of lg production by B-cells (Duhen et al., 2004), NK cell activation (Fan et al., 2006) and enhancement of the anti-bacterial activities of leukocytes (Heo et al., 2006). Researchers have reported that administration of an HVEM-lg fusion protein consisting of the soluble extracellular domain of HVEM linked to the immunoglobulin FC region affects HVEM-associated physiological functions such as mice immune responses to bacterial infections, the severity of graft-versus-host disease, collagen-induced arthritis, and cardiac allograft rejection (Ye et al., 2002; Pierer et al., 2009; Brown et al., 2010). Therefore, there is a good possibility that the SHVEM generated by ectodomain shedding has an in vivo role. Because HVEM has multiple ligands, such as LIGHT, BTLA and CD160, and LIGHT can bind to LT $\beta R$ in addition to HVEM,
sHVEM-mediated affects on molecular interactions could be very complex. Such interactions might include HVEM-LIGHT, HVEM-BTLA, HVEM-CD160 and LIGHT-LT $\beta R$ interactions. Identification of the role of the sHVEM might suggest ways to interfere with gastric cancer development.

The immune system can recognize and eliminate transformed tumor cells in a process named cancer immunosurveillance. However, the immune system can also promote tumor progression. These dual anti-tumor and pro-tumor actions of the immune system are now referred to as cancer immunoediting (Schreiber et al., 2011). Proinflammatory cytokines have major effects on the pro-tumor activities of the immune system. For example, TNF- $\alpha$ produced by tumor cells and leukocytes promotes the survival and proliferation of malignant cells, stimulates angiogenesis and metastasis, subverts adaptive immunity, and alters responses to hormones and chemotherapy (Balkwill, 2009). There is good evidence that immune cell-mediated inflammation also regulates the development and progression of gastric cancers. Thus, the major cause of gastric cancer is $H$. pylori infection, which is now recognized as an infectious disease because chronic $H$. pylori infection is so closely related to GC development (Piazuelo et al., 2010). Among 1526 Japanese patients who had gastric diseases, gastric cancer developed only in those patients infected by $H$. pylori (Uemura et al., 2001). Moreover, polymorphisms of several inflammatory cytokine genes, such as those for TNF- $\alpha$ and IL-1, affect GC risk (Crusius et al., 2008; Seo et al., 2010). In addition, GC patients have high levels of inflammatory cytokines in their sera as shown in this study (Figure 2) and by others (Tsujimoto et al., 2010). Although the role of HVEM in gastric cancer in association with inflammation is unknown, our finding that GC patient leukocytes have low levels of mHVEM but produce high levels of sHVEM adds to the evidence that GC is closely associated with inflammatory processes. Furthermore, it is noteworthy that in addition to gastric cancer, many cancers, such as hepatocellular carcinoma, B-cell lymphoma and cervical carcinoma are caused by infectious agents, which are closely associated with chronic inflammation (Coussens and Werb, 2002). Therefore, there is a substantial possibility that those cancer patients also have altered levels of sHVEM and MHVEM in their sera and leukocytes. We are currently investigating the level of sHVEM and mHVEM in various tumor patients. 


\section{Methods}

\section{Reagents}

Recombinant human LIGHT (rhLIGHT) was purchased from R\&D Systems (Minneapolis, MN), and diluted in $0.1 \%$ BSA-PBS buffer. According to the suppliers, the endotoxin concentration was less than $0.1 \mathrm{ng}$ per $1 \mu \mathrm{g}$ of protein as determined by the LDL method. Recombinant human IL-8 (rhIL-8), rhTNF- $\alpha$ and rhIL-2 proteins were purchased from Endogen (MA, USA). Polymorphprep ${ }^{\mathrm{TM}}$ for purifying neutrophils was from Nycomed Pharma (Oslo, Norway) and RPMI 1640 and Hank's balanced salt solution (HBSS), from GibcoBRL (Grand Island, N.Y.). ELISA kits for cytokines were purchased from Endogen (Thermo Fisher Scientific, Rockford, IL). All other reagents were from Sigma (St. Louis, MO). For heat inactivation, proteins were incubated for $20 \mathrm{~min}$ at $80^{\circ} \mathrm{C}$.

\section{Test samples}

Patients with GC and healthy volunteers were enrolled after obtaining informed consent, and the study was approved by the institutional review board of Ulsan University Hospital (\#09-30). Blood samples were obtained once from healthy volunteers and were collected from GC patients one day prior to the first chemotherapy treatment at a time when they were chemotherapy-naïve. All enrolled individuals were free from any evidence of infection.

\section{Preparation of monocytes and neutrophils}

Human monocytes and neutrophils were purified from the heparinized blood $(20 \mathrm{U} / \mathrm{ml}$ of heparin) of healthy donors and GC patients (Heo et al., 2006). Peripheral blood mononuclear cells (PBMCs) were isolated by centrifugation on a Ficoll Hypaque-1077 gradient. Primary CD14 ${ }^{+}$monocytes were separated from PBMCs by negative selection with $\mathrm{CD}_{14}{ }^{+}$(Miltenyi Biotec $\mathrm{GmbH}$, Bergisch Gladbach, Germany). Neutrophils were purified from adult peripheral blood by centrifugation on a Polymorphprep ${ }^{\mathrm{TM}}$ gradient as described previously (Heo et al., 2006). After two washes with HBSS without $\mathrm{Ca}^{2+}$ and $\mathrm{Mg}^{2+}$, cells were resuspended in the appropriate medium. Isolated monocytes and neutrophils were more than $95 \%$ pure as analyzed by flow cytometry and Giemsa staining of cytospin preparations (data not shown).

\section{Flow cytometry}

Monocytes (CD14 ${ }^{+}$cells) and neutrophils were incubated in $20 \% \mathrm{AB}$-serum in PBS at $4^{\circ} \mathrm{C}$ for 30 min. Subsequently, they were washed twice with FACS buffer (PBS containing $0.3 \% \mathrm{BSA}$ and $0.1 \% \mathrm{NaN}_{3}$ ) and incubated with FITC-conjugated anti-HVEM-FITC mAbs or FITC-conjugated isotype control mAbs (Mouse IgG1) at $4^{\circ} \mathrm{C}$ for $30 \mathrm{~min}$. Samples were analyzed by flow cytometry using a Becton Dickinson FACScan flow cytometer, and percentages of $\mathrm{mHVEM}^{+}$ cells were calculated with CELL-Quest software (BD Biosciences, San Jose CA).

\section{Cytokine ELISA}

Sera from patients with $\mathrm{GC}$ and healthy controls were harvested and frozen at $-80^{\circ} \mathrm{C}$. Cytokines were assayed with ELISA kits for IFN- $\gamma$, IL-1 $\beta$, IL-2, IL-4, IL-6, IL-8, IL-10, IL-12 and TNF- $\alpha$.

\section{Monocyte culture and ELISA assay for soluble HVEM}

Monocytes were purified from healthy controls and adjusted to $2 \times 10^{6} \mathrm{cells} / \mathrm{ml}$ in RPMI 1640 medium containing $10 \%$ FBS. They were cultured at $37^{\circ} \mathrm{C}$ in the presence of various concentrations of rhLIGHT, rhIL-8, rhTNF- $\alpha$, rhIL-2, PMA, ionomycin, LPS, PHA or A23187. After $24 \mathrm{~h}$, cell-free supernatants were collected by centrifugation and frozen at $-80^{\circ} \mathrm{C}$.

To measure sHVEM in sera and culture supernatants, sandwich ELISAs were employed using two anti-HVEM mAbs (108 and 122) recognizing different epitopes (Heo et al., 2007). A standard curve was constructed with HVEM-Fc as the antigen. ELISA plates were coated with $100 \mu \mathrm{g} /$ well of mAb108 $(1 \mu \mathrm{g} / \mathrm{ml})$ and incubated overnight at $4^{\circ} \mathrm{C}$. After incubation, plates were washed and blocked with PBS containing 4\% BSA. Samples diluted in PBS containing $4 \%$ BSA were added, and plates were incubated overnight at $4^{\circ} \mathrm{C}$. After washing, $100 \mu \mathrm{l} /$ well of biotin-labeled $\mathrm{mAb} 122$ at $1 \mathrm{\mu g} / \mathrm{ml}$ in 4\% BSA were added and incubation continued for $1 \mathrm{~h}$ at $37^{\circ} \mathrm{C}$. After addition of streptavidinHRP, plates were developed with TMB substrate solution (Endogen, Pierce Biotechnology, Rockford, CA), and absorbencies at $450 \mathrm{~nm}$ were determined. A standard curve constructed with HVEM-Fc (R\&D system, Minneapolis, MN) indicated that the sensitivity limit was $16 \mathrm{pg} / \mathrm{ml}$. HVEM concentrations in sera and supernatants were expressed as means \pm SEM of quadruplicate samples.

\section{Bactericidal activity}

Staphylococcus aureus (ATCC 56389; American Type Culture Collection, Manassas, VA.) was grown in nutrient broth (Becton Dickinson, Sparks, MD.) and Helicobacter pylori (ATCC 26695) was grown in Brucella broth (Becton Dickinson). Aliquots were frozen at $-80^{\circ} \mathrm{C}$ and thawed prior to use. Killing of bacteria was measured as previously described (Heo et al., 2006). Briefly, overnight cultures of $S$. aureus $\left(1 \times 10^{7}\right.$ cells $\left./ \mathrm{ml}\right)$ and $H$. pylori $\left(1 \times 10^{7}\right.$ cells $\left./ \mathrm{ml}\right)$ were opsonized by incubation with $0.1 \%$ gelatin (w/v) and $10 \%(\mathrm{v} / \mathrm{v})$ human $A B$ serum in HBSS. One $\mathrm{ml}$ of HBSS containing $1 \times 10^{7}$ opsonized bacteria was added to $1 \times$ $10^{7}$ cells $/ \mathrm{ml}$ of monocytes or neutrophils iln $100 \mu$ of HBSS and incubated at $37^{\circ} \mathrm{C}$ for 3 min with continuous rotation to promote phagocytosis. Non-ingested bacteria were discarded by differential centrifugation for $5 \mathrm{~min}$ at $1200 \mathrm{rpm}$, and cells containing ingested bacteria were cultured at $37^{\circ} \mathrm{C}$ for $10 \mathrm{~min}$ with slow rotation in the presence of rhLIGHT or heat inactivated rhLIGHT. Killing was stopped by spinning the cells on ice after addition of $1 \mathrm{ml}$ distilled water containing $0.01 \%$ BSA. Cell clumps were disrupted by vigorous vortexing, and the number of viable bacteria was determined by plating 10-fold serial dilutions on agar plates. The \% killing was calculated as follows: $\%$ killing = [1-(the number of viable bacteria at time $t /$ the number of 
viable bacteria at time 0$)] \times 100$.

\section{Measurement of reactive oxygen species (ROS) by FACS}

ROS generated by monocytes and neutrophils were assayed with the ROS-sensitive fluorescent dye, 2', 7'-dichlorofluorescin diacetate (DCF-DA). Cells were stained with $5 \mu \mathrm{M}$ DCF-DA for $15 \mathrm{~min}$ at $37^{\circ} \mathrm{C}$ in the dark. After incubation with DCF-DA, they were washed twice with PBS buffer and resuspended in FACs buffer. DCF-DA-stained monocytes were then treated with rhLIGHT for $45 \mathrm{~min}$. After washing, they were analyzed by flow cytometry (FACS Calibur; BD Biosciences, San Jose CA), and numbers of DCF-DA positive cells were calculated and displayed in the form of histograms (Bass et al., 1983).

\section{Statistics}

Differences between experimental groups were evaluated by Student's $t$ test. Results are representative of at least three independent experiments from different blood donors. Data are presented as means \pm SEM.

\section{Acknowledgements}

We thank Dr. Kwon Byeong $S$ for permission to use anti-HVEM antibodies. This work was supported by a National Research Foundation of Korea (NRF) grant funded by the Korean government (MEST) (BRL2009-0087350). The first three authors contributed equally to the work.

\section{References}

Aggarwal BB. Signaling pathways of the TNF superfamily: a double-edged sword. Nat Rev Immunol 2003;3:745-56

Arribas J, Borroto A. Protein ectodomain shedding. Chem Rev 2002;102:4627-38

Balkwill F. Tumour necrosis factor and cancer. Nat Rev Cancer 2009;9:361-71

Bass DA, Parce JW, Dechatelet LR, Szejda P, Seeds MC, Thomas M. Flow cytometeric studies of oxidative product formation by neutrophils: a graded response to membrane stimulation. J Immunol 1983;130:1910-7

Benucci M, Saviola G, Baiardi P, Manfredi M, Sarzi-Puttini P, Atzeni F. Efficacy and safety of leflunomide or methotrexate plus subcutaneous tumour necrosis factor-alpha blocking agents in rheumatoid arthritis. Int $\mathrm{J}$ Immunopathol Pharmacol 2011;24:269-74

Brown GR, Lane GW, Whittington BJ. Disparate role of LIGHT in organ-specific donor T cells activation and effector molecules in MHC class II disparate GVHD. J Clin Immunol 2010;30:178-84

Coussens LM, Werb Z. Inflammation and cancer. Nature. 2002;420:860-7

Crusius JB, Canzian F, Capella G, Pena AS, Pera G, Sala N, et al. Cytokine gene polymorphisms and the risk of adenocarcinoma of the stomach in the European Prospective Investigation Into Cancer and Nutrition (EPIC-EURGAST). Ann Oncol 2008;19:1894-902

del Rio ML, Lucas CL, Buhler L, Rayat G, Rodriguez-Barbosa JI. HVEM/LIGHT/BTLA /CD160 cosignaling pathways as targets for immune regulation. J Leukoc Biol 2010;87:223-35

Duhen T, Pasero C, Mallet F, Barbarat B, Olive D, Costello RT. LIGHT costimulates CD40 triggering and induces immunoglobulin secretion; a novel key partner in $T$ cell-dependent B cell terminal differentiation. Eur J Immunol 2004;34:3534-41

Fan Z, Yu P, Wang Y, Wang Y, Fu ML, Liu W, Sun Y, Fu YX. NK-cell activation by LIGHT triggers tumor-specific CD8+ T-cell immunity to reject established tumors. Blood 2006;107:1342-51

Fortini ME. Notch and presenilin: a proteolytic mechanism emerges. Curr Opin Cell Biol 2001;13:627-34

Heo SK, Ju SA, Lee SC, Park SM, Choe SY, Kwon B, Kwon BS, Kim BS. LIGHT enhances the bactericidal activity of human monocytes and neutrophils via HVEM. J Leukoc Biol 2006; $79: 330-8$

Heo SK, Yoon MA, Lee SC, Ju SA, Choi JH, Suh PG, Kwon BS, Kim BS. HVEM signaling in monocytes is mediated by intracellular calcium mobilization. J Immunol 2007;179: 6305-10

Hikita A, Tanaka N, Yamane S, Ikeda Y, Furukawa H, Tohma S, Suzuki R, Tanaka S, Mitomi H, Fukui N. Involvement of a disintegrin and metalloproteinase 10 and 17 in shedding of tumor necrosis factor-alpha. Biochem Cell Biol 2009;87: 581-93

Jung, HW, La SJ, Kim JY, Heo SK, Kim JY, Wang S, Kim KK, Lee KM, Cho HR, Lee HW, Kwon B, Kim BS, Kwon BS. High levels of soluble herpes virus entry mediator in sera of patients with allergic and autoimmune diseases. Exp Mol Med 2003;35:501-8

Kwon BS, Tan KB, Ni J, Oh KO, Lee ZH, Kim KK, Kim YJ, Wang S, Gentz R, Yu GL, Harrop J, Lyn SD, Silverman C, Porter TG, Truneh A, Young PR. A newly identified member of the tumor necrosis factor receptor superfamily with a wide tissue distribution and involvement in lymphocyte activation. J Biol Chem 1997;272:14272-6

Mantovani A, Allavena P, Sica A, Balkwill F. Cancer-related inflammation. Nature 2008;454:436-44

Morel Y, Schiano de Colella JM, Harrop J, Deen KC, Holmes SD, Wattam TA, Khandekar SS, Truneh A, Sweet RW, Gastaut JA, Olive D, Costello RT. Reciprocal expression of the TNF family receptor herpes virus entry mediator and its ligand LIGHT on activated T cells: LIGHT down-regulates its own receptor. J Immunol 2000;165:4397-404

Piazuelo MB, Epplein M, Correa P. Gastric cancer: an infectious disease. Infect Dis Clin North Am 2010;24:853-69

Pierer M, Schulz A, Rossol M, Kendzia E, Kyburz D, Haentzschel H, Baerwald C, Wagner U. Herpes virus entry mediator-Ig treatment during immunization aggravates rheumatoid arthritis in the collagen-induced arthritis model. 
$\mathrm{J}$ Immunol 2009;182:3139-45

Rose-John S, Heinrich PC. Soluble receptors for cytokines and growth factors: generation and biological function. Biochem J 1994;300:281-90

Schreiber RD, Old LJ, Smyth MJ. Cancer immunoediting: integrating immunity's roles in cancer suppression and promotion. Science 2011;331:1565-70

Segal AW. How neutrophils kill microbes. Annu Rev Immunol. 2005;23:197-223

Seo GS, Lee JK, Yu JI, Yun KJ, Chae SC, Choi SC. Identification of the polymorphisms in IFITM3 gene and their association in a Korean population with ulcerative colitis. Exp Mol Med 2010;42:99-104

Shui JW, Steinberg MW, Kronenberg M. Regulation of inflammation, autoimmunity, and infection immunity by HVEM-BTLA signaling. J Leukoc Biol 2011;89:517-23

Tamada K, Shimozaki K, Chapoval Al, Zhai Y, Su J, Chen SF, Hsieh SL, Nagata S, Ni J, Chen L. LIGHT, a TNF-like molecule, costimulates $T$ cell proliferation and is required for dendritic cell-mediated allogeneic $\mathrm{T}$ cell response. J Immunol 2000;164:4105-10

Tsujimoto H, Ono S, Ichikura T, Matsumoto Y, Yamamoto J, Hase K. Roles of inflammatory cytokines in the progression of gastric cancer: friends or foes? Gastric Cancer 2010;13: 212-21

Uemura N, Okamoto S, Yamamoto S, Matsumura N,
Yamaguchi S, Yamakido M, Taniyama K, Sasaki N, Schlemper RJ. Helicobacter pylori infection and the development of gastric cancer. N Engl J Med 2001;345: 784-89

Wallach D, Varfolomeev EE, Malinin NL, Goltsev YV, Kovalenko AV, Boldin MP. Tumor necrosis factor receptor and Fas signaling mechanisms. Annu Rev Immunol 1999;17:331-67

Wong ST, Winchell LF, McCune BK, Earp HS, Teixidó J, Massagué J, Herman B, Lee DC. The TGF-alpha precursor expressed on the cell surface binds to the EGF receptor on adjacent cells, leading to signal transduction. Cell 1989;56: 495-506

Wroblewski LE, Peek RM Jr, Wilson KT. Helicobacter pylori and gastric cancer: factors that modulate disease risk. Clin Microbiol Rev 2010;23:713-39

Ye Q, Fraser CC, Gao W, Wang L, Busfield SJ, Wang C, Qiu Y, Coyle AJ, Gutierrez-Ramos JC, Hancock WW. Modulation of LIGHT-HVEM costimulation prolongs cardiac allograft survival. J Exp Med 2002;195:795-800

Zhu LJ, Yang X, Yu XQ. Anti-TNF-alpha therapies in systemic lupus erythematosus. J Biomed Biotechnol 2010;2010: 465898

Zou GM, Martinson J, Hu WY, Tam Y, Klingemann HG. The effect of LIGHT in inducing maturation of monocyte-derived dendritic cells from MDS patients. Cancer Immunol Immunother 2004;53:681-9 